\title{
Universality classes and crossover behaviors in non-Abelian directed sandpiles
}

\author{
Hang-Hyun $\mathrm{Jo}^{1, \text {, }}$ and Meesoon $\mathrm{Ha}^{2,1, \text {, }}$ \\ ${ }^{1}$ School of Physics, Korea Institute for Advanced Study, Seoul 130-722, Korea \\ ${ }^{2}$ Department of Physics, Korea Advanced Institute of Science and Technology, Daejeon 305-701, Korea
}

(Dated: November 21, 2018)

\begin{abstract}
We study universality classes and crossover behaviors in non-Abelian directed sandpile models, in terms of the metastable pattern analysis. The non-Abelian property induces spatially correlated metastable patterns, characterized by the algebraic decay of the grain density along the propagation direction of an avalanche. Crossover scaling behaviors are observed in the grain density due to the interplay between the toppling randomness and the parity of the threshold value. In the presence of such crossovers, we show that the broadness of the grain distribution plays a crucial role in resolving the ambiguity of the universality class. Finally, we claim that the metastable pattern analysis is important as much as the conventional analysis of avalanche dynamics.
\end{abstract}

PACS numbers: 05.70.Ln, 05.65.+b, 64.60.Ht

\section{INTRODUCTION}

Scale invariance in avalanche systems is ubiquitously observed in nature, such as earthquakes, sandpiles, and Barkhausen avalanches of ferromagnetic materials. The statistics of avalanches follows some universal powerlaw distribution [1-3]. Metastable patterns between avalanches also exhibit spatially long-range correlations. A fractal structure in the crust of the earth formed by seismic events is one of such examples. It is, however, still questionable to find generic mechanisms for avalanche systems that can explain both the statistics of avalanches and long-range correlations in metastable patterns.

To reveal the underlying common mechanism of scale invariance in avalanche systems, Bak, Tang, and Wiesenfeld (BTW) first proposed the paradigm of self-organized criticality with an Abelian deterministic version of undirected sandpile models. Since then, lots of BTW variants have been suggested and studied [4 $[6]$. In modeling sandpiles, the balance between slow driving and dissipation is the key ingredient. Grains are slowly added, toppled instantly whenever the instability threshold is overcome, and finally dissipated at boundaries of the system. It has been tested whether the universality class of avalanche dynamics can be changed by the breaking of Abelian symmetry [7] or the consideration of stochasticity [8] in toppling rules. The issue is still controversial due to conflicting numerical results $9-13$ ]. The Abelian symmetry here means that the order of toppling events does not affect the final state.

Contrary to undirected sandpile models, directed sandpile models (DSMs) with a preferred direction of avalanche propagation turn out to be more tractable analytically as long as they have the Abelian symmetry 1417]. This is because the Abelian symmetry in DSMs lets

*Present address: BECS, School of Science and Technology, Aalto University, P.O. Box 12200, FI-00076

${ }^{\dagger}$ Corresponding author: msha@kaist.ac.kr metastable grain patterns be fully uncorrelated. However, most real avalanche systems often exhibit spatially correlated grain patterns. It means that Abelian DSMs are not suitable enough to describe such systems and non-Abelian DSMs are more natural to be considered.

When the Abelian symmetry is broken in DSMs 18 20], spatially long-range correlations emerge in metastable grain and scar patterns. Scars are defined as traces of avalanche boundaries. Both scar and grain densities decay algebraically along the preferred direction of avalanche propagation with the same decay exponent. In our earlier work [20], additional scaling relations are derived from avalanche flow equations. All avalanche exponents can be written in terms of the grain density exponent. With the stochastic toppling rule, the broken Abelian symmetry seems not to change the universality class. However, it changes the scaling behavior of metastable patterns with the non-zero value of the grain density exponent. When the toppling rule becomes deterministic, the Abelian symmetry turns out to be relevant to the universality class and non-Abelian DSMs belong to the mean-field (MF) universality class. These results were numerically confirmed in the simplest models [20].

However, the simplest discrete DSM with the nonAbelian deterministic toppling rule cannot be unique due to the interplay between the toppling rule and the lattice structure. For an example, if the number of grains at an unstable site is 3 and the number of its neighboring sites is 2 , there are several ways to topple the last grain into one of the neighboring sites. Two versions of the toppling bias for the last grain were considered, which lead to some deviation from MF results [20].

In this paper, we check out the validity of universality classes in non-Abelian DSMs against the change of the toppling bias for the last grain and the instability threshold value. We also report various crossover behaviors between universality classes. One might expect that the toppling bias effect becomes negligible as the threshold value increases. It turns out to be only true for the fully biased case. For alternatively and/or partially bi- 
ased cases, the parity of the threshold value plays a crucial role in avalanche dynamics. If the threshold value is odd, the stochasticity in toppling rules becomes relevant. As a result, the deterministic models belong to the nonAbelian stochastic universality class. It is somehow surprising because one believes the threshold value in sandpile models to be irrelevant to universality classes, except for crossover behaviors discussed in undirected cases by Lübeck 21].

This paper is organized as follows. In Sec. III we briefly describe various DSMs including those proposed in 20]. In Sec. III, we discuss the role of the threshold value and the toppling bias in determining universality classes by the conventional analysis of avalanche dynamics and the metastable pattern analysis. We also argue possible scenarios for universality classes and crossover behaviors, which are numerically confirmed in Sec. IV] Finally, we conclude this paper in Sec. $\mathrm{V}$ with the summary of main results and some suggestions.

\section{DIRECTED SANDPILE MODELS}

We consider DSMs defined on a two-dimensional $\frac{\pi}{4}$ rotated lattice of sizes $\left(L_{\perp}, L_{\|}\right)$. For convenience, we call $L_{\perp}$ and $L_{\|}$as $L$ and $T$, respectively. The preferred direction of avalanche propagation is denoted by the "layer" $t=0,1, \ldots, T-1$ with open boundary conditions. The transverse direction is denoted by $i=0,1, \cdots, L-1$ with periodic boundary conditions (see Fig. 1). Initially, to each site of the lattice an integer value (the number of grains), $z(i, t) \in\left\{0,1, \ldots, z_{c}-1\right\}$, is assigned. Here $z_{c}$ denotes the threshold value.

Given a stable configuration where $z(i, t)<z_{c}$ for all sites $(i, t)$, one grain is added at a randomly chosen site on the top layer, $z(i, 0) \rightarrow z(i, 0)+1$. For any unstable site with $z(i, t) \geq z_{c}$, including that on the top layer, grains topple down to its left and right nearest-neighboring sites



FIG. 1: A two-dimensional $\frac{\pi}{4}$-rotated lattice for DSMs.
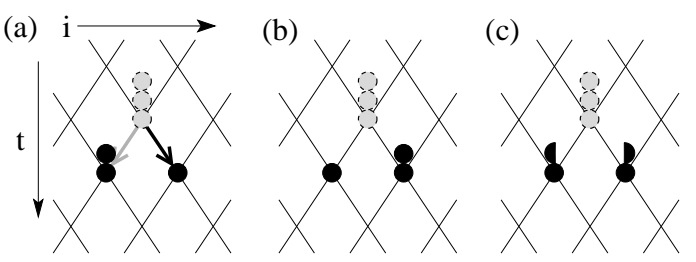

FIG. 2: Toppling rules of non-Abelian deterministic DSMs on a two-dimensional tilted lattice [20]: (a) aND, (b) bND, and (c) cND. The gray colored grains and arrow represent the state before toppling and the black colored ones represent the state after toppling.

on the next layer $t+1$.

$$
\begin{aligned}
z(i, t) & \rightarrow z(i, t)-\Delta_{i, t}, \\
z(i \pm 1, t+1) & \rightarrow z(i \pm 1, t+1)+\tilde{\Delta}_{i \pm 1, t+1} .
\end{aligned}
$$

Here $\Delta_{i, t}$ represents the number of grains outgoing from $(i, t)$ and $\tilde{\Delta}_{i \pm 1, t+1}$ does the number of grains incoming to $(i \pm 1, t+1)$ from $(i, t)$. The number of toppled grains is locally conserved: $\Delta_{i, t}=\tilde{\Delta}_{i-1, t+1}+\tilde{\Delta}_{i+1, t+1}$.

The toppling at one layer may cause another toppling on the next layer. At unstable sites on the bottom layer $t=T-1$, toppled grains are dissipated out of the system. Only after another stable configuration is recovered by a series of toppling events, denoting an avalanche, a new grain is added on the top layer, $t=0$, to keep generating another avalanche.

By setting the toppling matrices, $\Delta$ and $\tilde{\Delta}$, one may consider several variants of DSMs. Without losing any generality, we set $\Delta_{i, t}=z_{c}$ in the Abelian version and $\Delta_{i, t}=z(i, t) \geq z_{c}$ in the non-Abelian version. The values of $\tilde{\Delta}_{i \pm 1, t+1}$ can be either stochastically or deterministically determined. For the stochastic case, each grain at the unstable site is toppled at random to one of the nearest neighbors at the next layer. Abelian and nonAbelian stochastic DSMs are denoted as AS and NS for short, respectively. For Abelian deterministic DSMs (AD for short), one should fix the values of $\tilde{\Delta}_{i \pm 1, t+1}$ under the condition $\tilde{\Delta}_{i-1, t+1}+\tilde{\Delta}_{i+1, t+1}=z_{c}$. For an example, each value of $\tilde{\Delta}_{i \pm 1, t+1}$ is set as one half of $z_{c}$ when $z_{c}$ is even.

For non-Abelian deterministic DSMs (ND for short) where the simplest setup is not clear, the following three versions are considered in [20]:

$$
\begin{aligned}
\text { (i) } \tilde{\Delta}_{i \pm 1, t+1} & = \begin{cases}k & \text { if } z(i, t)=2 k \\
k+\delta_{i \pm 1, a(i, t)} & \text { if } z(i, t)=2 k+1,\end{cases} \\
\text { (ii) } \tilde{\Delta}_{i \pm 1, t+1} & = \begin{cases}k & \text { if } z(i, t)=2 k \\
k+\delta_{i \pm 1, i+1} & \text { if } z(i, t)=2 k+1,\end{cases} \\
\text { (iii) } \tilde{\Delta}_{i \pm 1, t+1} & =z(i, t) / 2 .
\end{aligned}
$$

Here, $k$ is a positive integer and $\delta_{i j}$ denotes the Kronecker delta function. We call (i) the alternative bias version (aND) 19], (ii) the full bias version (bND), and (iii) the continuous version without bias (cND). For the aND, a 
(a)

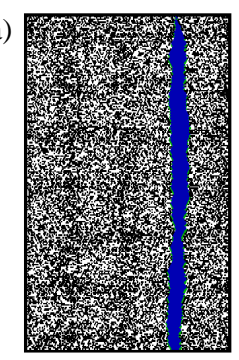

(b)

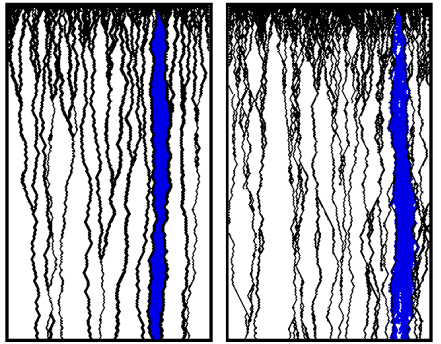

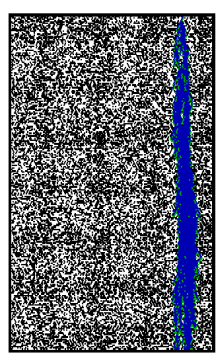
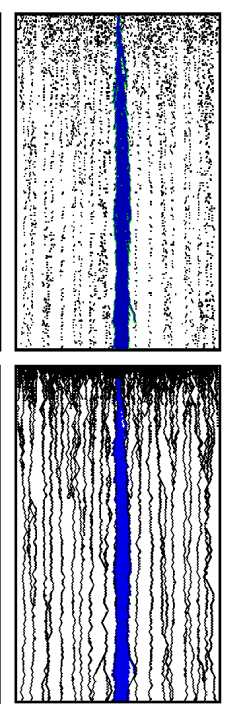


FIG. 3: (Color online) Typical metastable grain (a) patterns and (b) scars of six DSMs (from left to right, AD, AS, NS, aND, $\mathrm{bND}$, and $\mathrm{cND})$ at $z_{c}=2$ : occupied sites and scars are shown as black dots and the typical shapes of dissipative avalanches consisting of toppled sites as blue (shaded) areas on a lattice with $L=150$ (horizontally) and $T=250$ (vertically).

"toppling arrow" $a(i, t)$ of each site initially points to one of its neighbors, say, $i-1$, in Fig. 2(a). Whenever each grain is toppled at the unstable site, the direction of the arrow flips to the other neighbor. Figure 2 shows the case of $z(i, t)=3$ and $z_{c}=2$. It is worthwhile to note that any toppling bias here is applied only to the last grain when the number of toppled grains at the unstable site, $z(i, t)$, is odd.

In order to check the validity of earlier ND versions, we introduce another ND version that covers both the aND and the bND by controlling the bias of the last grain at the toppled site as follows:

$$
\text { (iv) } \tilde{\Delta}_{i \pm 1, t+1}= \begin{cases}k & \text { if } z(i, t)=2 k \\ k+\delta_{i \pm 1, r(i, t)} & \text { if } z(i, t)=2 k+1\end{cases}
$$

Here $r(i, t)$ takes $i+1$ with a probability $p$ or $i-1$ with a probability $1-p$ for each toppling event. For convenience, only the range of $\frac{1}{2} \leq p \leq 1$ is considered. We call (iv) the partial bias version (pND for short). This model is exactly the same as the bND when $p=1$. Moreover, it is almost the same as the aND when $p=\frac{1}{2}$, except that toppling arrows are initially quenched in the aND or completely annealed in the pND.

An avalanche of DSMs can be quantified as mass $s$ (the total number of toppled grains), duration $t$ (the number of affected layers), area $a$ (the total number of distinct toppled sites), width $w$ (the distance between avalanche boundaries), and height $h$ (the number of toppled grains per toppled site). There is no characteristic scale in avalanche dynamics, except for $T$ as long as $L$ is sufficiently larger than the maximum width of the avalanche. It is well known that all probability distribution functions of avalanche quantities are expected to follow the simple scaling form as

$$
P(x, T) \sim x^{-\tau_{x}} f\left(x / T^{D_{x}}\right)
$$

for $x \in\{s, t, a, w, h\}$. Hence, any two quantities $x$ and $y$ scale, using the conditional expectation value, as

$$
E[y \mid x] \sim x^{\gamma_{y x}} .
$$

The relative exponent $\gamma_{y x}=\left(\tau_{x}-1\right) /\left(\tau_{y}-1\right)=D_{y} / D_{x}$ is derived from the identity $P(x, T) d x=P(y, T) d y$.

We take full advantage of the relations, $D_{t}=1$ and $\langle s\rangle \sim T$ in DSMs, where $\langle\cdot\rangle$ denotes the ensemble average. Based on the reasonable assumption for the compactness of the avalanche in low dimensional systems, i.e., $a \sim w t$ and $s \sim a h$, we obtain the following scaling relations:

$$
\begin{array}{r}
\gamma_{x t}=D_{x} \text { for any } x, \\
D_{s}\left(2-\tau_{s}\right)=1, \\
D_{a}=D_{w}+1, \text { and } D_{s}=D_{a}+D_{h}
\end{array}
$$

which implies only two independent exponents left.

Concerning metastable patterns of DSMs, we are able to define two additional scaling exponents. In order to quantify metastable patterns, we denote the probability to find $z$ remaining grains at each layer $t$ as

$$
Q(z, t) \equiv \operatorname{Pr}[z(i, t)=z]
$$

where $\sum_{z=0}^{z_{c}-1} Q(z, t)=1$. Along the avalanche propagation direction, one can measure the grain density as

$$
\rho(t) \equiv \frac{1}{L} \sum_{i=0}^{L-1}\langle z(i, t)\rangle=\sum_{z=0}^{z_{c}-1} z Q(z, t),
$$

where $\langle\cdot\rangle$ denotes the recurrent configuration average. Similarly, the fraction of occupied sites at each layer, 
namely, the grain occupation density, can be measured as

$$
\rho_{\theta}(t) \equiv \frac{1}{L} \sum_{i=0}^{L-1}\langle\theta[z(i, t)]\rangle=\sum_{z=1}^{z_{c}-1} Q(z, t),
$$

where $\theta(z)$ denotes the Heaviside step function that gives 0 for $z=0$ and 1 for $z>0$. Both $\rho(t)$ and $\rho_{\theta}(t)$ decay algebraically with the grain density exponent $\alpha$ at large $t$ in non-Abelian DSMs as

$$
\rho(t) \sim \rho_{\theta}(t) \sim t^{-\alpha}
$$

For Abelian DSMs with an uniform grain density $(\alpha=0)$, we consider the scar density $\rho_{\mathrm{sc}}(t)$ and the scar exponent $\alpha_{\mathrm{sc}}$ with the same definition of $\rho(t)$ where $z(i, t)$ is just replaced by the quantity, $b(i, t)$. The value of $b(i, t)$ is 1 if the site $(i, t)$ has recently been a part of avalanche boundaries, or 0 otherwise. We call $\{b(i, t)\}$ a scar.

\section{AVALANCHE DYNAMICS}

We begin with the discussion of statistical properties observed in metastable grain and scar patterns. The metastable pattern analysis is important as much as the conventional analysis of avalanche dynamics. As reported in [20], the scar exponent is a good indicator for the universality classes of DSMs since it is directly related to the exponent of the avalanche width as $\alpha_{\mathrm{sc}}=D_{w}$. It is because the scar density is inversely proportional to the typical avalanche width.

$$
\rho_{\mathrm{sc}}(t)=\frac{1}{L} \sum_{i=0}^{L-1}\langle b(i, t)\rangle \approx \frac{1}{L} \frac{L}{w(t)}=w(t)^{-1} .
$$

It is worthwhile to note that grains in non-Abelian DSMs can remain only at avalanche boundaries, which implies $\alpha=\alpha_{\text {sc }}$. On the other hand, Abelian DSMs exhibit an uncorrelated and uniform grain density in metastable grain patterns, i.e., $\alpha=0$, irrespective of the $\alpha_{\mathrm{sc}}$ value.

We intuitively argue the interplay between avalanche flow and metastable grain or scar patterns in DSMs. Let us define $N(t)$ as the number of grains toppled from the layer $t$ to the next layer $t+1$ within an avalanche. It scales as $N(t) \sim w(t) h(t) \sim t^{D_{w}+D_{h}}$ with the assumption for the compactness of an avalanche. The evolution of $N(t)$, avalanche flow, can be written as

$$
\frac{d N(t)}{d t} \approx N(t)-N(t-1)=\sum_{i \in w(t)} n(i, t),
$$

where $n(i, t)$ denotes the amount of avalanche flow at the site $(i, t)$. The value of $n(i, t)$ depends on both the value of $z(i, t)$ before toppling and the number of grains toppled from the previous layer to the site $(i, t)$. The summation is over avalanche boundaries and bulk sites, belonging to $w(t)$. Here avalanche boundaries denote the outermost sites of an avalanche at a given layer and avalanche bulk sites do the sites between avalanche boundaries.

\section{A. Abelian cases}

In AD DSMs, it is well known that metastable grain patterns are fully uncorrelated and that probabilities to find $z(i, t) \in\left\{0,1, \ldots, z_{c}-1\right\}$ grains at a site are the same as $1 / z_{c}$ [14]. The number of toppled grains at every unstable site is $z_{c}$, such that $D_{h}=0$. Since $n(i, t)=0$ at avalanche bulk sites, only avalanche boundaries contribute to avalanche flow. At the right avalanche boundary, the avalanche width $w$ increases by one if the toppling at the rightmost site of the avalanche, say $(i, t)$, leads to a toppling at a site $(i+1, t+1)$. The probability is given by

$$
\operatorname{Pr}\left[z(i+1, t+1)+\tilde{\Delta}_{i+1, t+1} \geq z_{c}\right]=\frac{\tilde{\Delta}_{i+1, t+1}}{z_{c}} \equiv p_{\mathrm{AD}} .
$$

The probability to decrease $w$ by 1 is $1-p_{\mathrm{AD}}$. Likewise, probabilities to increase and decrease $w$ by one at the left avalanche boundary are $1-p_{\mathrm{AD}}$ and $p_{\mathrm{AD}}$, respectively. For the range of $0<p_{\mathrm{AD}}<1$, avalanche boundaries in the $\mathrm{AD}$ are exactly mapped onto the spatiotemporal trajectories of random walks starting at the same site. The avalanche flow equation is therefore described as

$$
\frac{d N(t)}{d t} \approx \eta(t)
$$

where $\eta(t)$ denotes an uncorrelated noise with zero mean and unit variance. As a result, we obtain $\alpha_{\mathrm{sc}}=D_{w}=\frac{1}{2}$ with $D_{h}=0$, which yields all avalanche exponent values. We note that the result in the AD is robust, independent of the value of $z_{c}$ and any possible toppling bias in the case of $p_{\mathrm{AD}} \neq \frac{1}{2}$.

In AS DSMs, the fluctuations of $\tilde{\Delta}_{i \pm 1, t+1}$ resulting from the stochastic toppling rule enable multiple topplings (more than one toppling at the same site). Metastable grain patterns are still fully uncorrelated because of the characteristic of the Abelian symmetry $(\alpha=0)$ [16, 17]. In that sense, avalanche boundaries in the AS are also mapped onto the trajectories of random walks, $\alpha_{\mathrm{sc}}=D_{w}=\frac{1}{2}$. However, avalanche flow in the AS is mainly affected by avalanche bulk sites. In general, the numbers of grains that bulk sites receive from the previous layer can be written as $m z_{c}+l$, where $m$ is a non-negative integer and $0 \leq l \leq z_{c}-1$. If $z(i, t)+l<z_{c}$ with the value of $z(i, t)$ in the metastable state, $l$ grains would be left behind avalanche flow, i.e., $n(i, t)=-l$. If $z(i, t)+l \geq z_{c}$, avalanche flow would sweep $z_{c}-l$ grains, i.e., $n(i, t)=z_{c}-l$. Based on the reasonable assumption that the probability distribution of $l$ is uniform, $n(i, t)$ is considered as a random variable $\eta(i, t)$ with zero mean and finite variance. As a result, the avalanche flow equation in the AS is described as

$$
\frac{d N(t)}{d t} \approx \sum_{i \in w(t)} \eta(i, t) \approx \sqrt{w(t)} \eta(t)
$$

which gives $D_{h}=\frac{1}{4}$. A positive value of $D_{h}$ reflects the existence of the multiple toppling. Once again, above 
results are also robust, independent of the value of $z_{c}$. The toppling bias can be considered as setting the probabilities of each grain toppled to the right and the left nearest neighbors at the next layer with $p_{\mathrm{AS}}$ and $1-p_{\mathrm{AS}}$, respectively. In other words,

$$
p_{\mathrm{AS}}=\frac{\left\langle\tilde{\Delta}_{i+1, t+1}\right\rangle}{z_{c}}
$$

where $\langle\cdot\rangle$ denotes the ensemble average due to the stochasticity in toppling rules. When $0<p_{\mathrm{AS}}<1$, such toppling bias does not change the scaling properties of avalanche dynamics (numerically confirmed but not shown here).

\section{B. Non-Abelian cases}

In NS DSMs, spatially correlated metastable patterns are well described as the algebraic decay of the grain density along the avalanche propagation direction [18]. Due to such spatial correlations in metastable patterns, the uncorrelated noise $\eta$ in the avalanche flow equation for Abelian cases should be replaced by some correlated noise to represent NS metastable patterns. Naively speaking, one can interpret $\eta$ in Eq. (14) as $\rho_{\mathrm{sc}}$ having the same dimension of $t^{-1 / 2}$ as $\eta$. Thus, we replace $\rho_{\text {sc }}$ by $\rho$ in the NS, while keeping $\sqrt{w(t)}$ due to the stochasticity in toppling rules:

$$
\frac{d N(t)}{d t} \approx \sqrt{w(t)} \rho(t)
$$

which yields $D_{h}=1-\frac{3}{2} \alpha$. As a result all other avalanche exponents in the NS can be written in terms of $\alpha$, such as $\tau_{s}=\frac{2(3-\alpha)}{4-\alpha}$ and $\tau_{t}=2-\frac{\alpha}{2}$.

The value of $\alpha$ in the $\mathrm{NS}^{2}$ is numerically observed as 0.45 , slightly less than $\frac{1}{2}$. In the earlier work [20], it is argued that the deviation seems to be attributed to logarithmic corrections to scaling. So we simply assume the random walk behaviors of avalanche boundaries, such that $\alpha=\frac{1}{2}$, irrespective of the toppling bias and the threshold value (numerically confirmed but not shown here). Then, all avalanche exponents in the NS becomes exactly the same as those in the AS: $\tau_{s}=\frac{10}{7}, \tau_{t}=\frac{7}{4}$, and $D_{h}=\frac{1}{4}$. From now on, we call the set of these exponents as the "NS" universality class. One can say that the NS belongs to the same universality class of the AS in the following sense: for Abelian cases, avalanche flow can sweep and lose many grains at the same time due to the uniform grain density. On the other hand, for non-Abelian cases, avalanche flow can sweep only few grains due to the power-law decaying grain density, but it can also leave only few grains behind as taking all grains at unstable sites. For both cases, the scaling property of $N(t)$ is apparently unaffected by the grain density as long as the toppling rule is stochastic. Finally, we naively propose another possible scenario for $\alpha=\frac{1}{2}$ in
TABLE I: Avalanche exponents $\left\{\tau_{s}, \tau_{t}, D_{h}\right\}$, grain density and scar exponents, $\alpha$ and $\alpha_{\mathrm{sc}}$ and our conjecture in twodimensional DSMs: the error bars of numerical results are obtained from effective exponent plots by the assumption of the dominant simple power-law scaling [23]. It is worthwhile to note that $D_{s}=\tau_{t}$ and $D_{t}=1$ in all DSMs and $\alpha_{\mathrm{sc}}=\alpha$ in non-Abelian DSMs.

\begin{tabular}{lcccc}
\hline \hline Model & $\tau_{s}$ & $\tau_{t}$ & $D_{h}$ & $\alpha, \alpha_{\mathrm{sc}}$ \\
\hline Mean field $\left(d_{u}=2\right)$ & $\frac{3}{2}$ & 2 & 0 & \\
& & & & \\
Abelian & & & & \\
$\quad$ Deterministic & $\frac{4}{3}$ & $\frac{3}{2}$ & 0 & $0, \frac{1}{2}$ \\
Stochastic & $\frac{10}{7}$ & $\frac{7}{4}$ & $\frac{1}{4}$ & $0, \frac{1}{2}$ \\
& & & & \\
Non-Abelian & & & & \\
Stochastic & $\frac{2(3-\alpha)}{4-\alpha}$ & $2-\frac{\alpha}{2}$ & $1-\frac{3 \alpha}{2}$ & $\alpha$ \\
$-z_{c}=2$ & $1.43(1)$ & $1.78(1)$ & $0.31(3)$ & $0.45(3)$ \\
& & & & \\
Deterministic & $\frac{3}{2}$ & 2 & $1-\alpha$ & $\alpha$ \\
aND & & & & \\
$-z_{c}=2$ & $1.49(1)$ & $1.94(1)$ & $0.07(1)$ & $0.86(3)$ \\
$-z_{c}=3$ & $1.46(2)$ & $1.78(3)$ & $0.26(1)$ & $0.37(1)$ \\
$-z_{c}=4$ & $1.53(1)$ & $2.00(5)$ & $0.16(2)$ & $0.97(1)$ \\
$\mathrm{bND}$ & & & & \\
$-z_{c}=2$ & $1.43(1)$ & $1.79(1)$ & $0.06(1)$ & $0.69(5)$ \\
$-z_{c}=3$ & $1.46(2)$ & $1.85(3)$ & $0.20(2)$ & $0.78(14)$ \\
$-z_{c}=4$ & $1.51(2)$ & $2.02(3)$ & $0.15(1)$ & $0.89(15)$ \\
$\mathrm{cND}$ & & & & \\
$-z_{c}=2$ & $1.52(3)$ & $2.04(3)$ & $0.06(4)$ & $0.91(11)$ \\
\hline \hline
\end{tabular}

the NS by mapping metastable patterns onto the $(1+1)$ dimensional spatiotemporal configuration of $2 A \rightarrow A$ coagulation-diffusion model, where the particle density temporally decays as $t^{-1 / 2}$ 22]. However, this scenario requires further investigation.

In ND DSMs, there are no fluctuations if we focus on the continuous version, namely the $\mathrm{cND}$, shown in Fig. 2(c). The avalanche flow equation can be written as

$$
\frac{d N(t)}{d t} \approx w(t) \rho(t)
$$

yielding $D_{h}=1-\alpha$. We find that the scaling exponents of avalanche mass and duration are MF values, independent of $\alpha$, i.e., $\tau_{s}=\frac{3}{2}$ and $\tau_{t}=2$, whereas other exponents still depend on $\alpha$. In that sense, we point out that one should check other avalanche exponents besides those of the avalanche mass and duration to discuss the universality class in the ND. If the assumption of $\alpha=D_{w}=1$ is accepted based on the linear behavior of avalanche boundaries, all avalanche exponents turn to be MF values, except for the case of the avalanche width 24]. Furthermore, this may also correspond to the MF behavior of the $(d+1)$-dimensional spatiotemporal configuration of the coagulation-diffusion model when $d \geq d_{u}=2$, where the particle density decays as $t^{-1}$ [22]. The peculiar "MF" behavior in the two-dimensional ND, i.e., appeared in the low dimensional system, can be easily understood in the context of the shape of $N(t)$ with its width and height. 
Toppling rules we considered in the ND suppress the fluctuations of the height profile in avalanche flow much more than those in the NS do. This leads to spread grains wider and wider and makes avalanche boundaries grow faster, almost ballistically. Such a positive feedback enables $D_{w}=1$ to be larger than $\frac{1}{2}$ for all other DSMs. The resultant $D_{h}=0$ indicates the MF behavior with $\alpha=1$, whereas $D_{h}=0$ for any dimensions in the AD.

Table I presents the avalanche exponents, the grain density exponent, and the scar exponent, as well as the exact results for the Abelian case and our conjecture for the non-Abelian case.

We report that the numerical results in the aND and the bND clearly deviate from MF results. As the possible origin of anomalous scaling behaviors, we claim that they are attributed to the toppling bias applied to the last grain when $z(i, t)=2 k+1 \geq z_{c}$ in Eq. (2). The toppling bias effect in the ND is numerically tested as $z_{c}$ increases. We find that the increment of $z_{c}$ may yield either that the toppling bias becomes negligible compared to other $(2 k)$ grains, or that it turns to be relevant to change the universality class. The aND with even $z_{c}$ values and the bND with all $z_{c}$ values correspond to the former. They show the MF behavior with some logarithmic corrections for small $z_{c}$ values and without such corrections for large $z_{c}$ values as expected (see Table \). Scaling behaviors in the aND with odd $z_{c}$ values are clearly deviated from MF results, and seem to be those of the NS class.

Two different mechanisms can be illustrated for the MF class and the NS class emerged in the aND against the parity of $z_{c}$. After the transient period of sandpile models, each site having ever toppled should be empty or occupied by at least $\left[z_{c} / 2\right]$ grains. Here $[m]$ is the integer value that is smaller than or equal to $m$.

In the even case with $z_{c}=2 k$, at least $k$ grains at the rightmost toppled site of the avalanche, say, $(i, t)$, topple to its right neighboring site on the next layer, $(i+1, t+1)$. The site $(i+1, t+1)$ will topple if occupied by grains, or will not if empty. At small $t$, the grain density is relatively high and the avalanche width is still small. Grains swept by avalanche flow quickly diffuse to avalanche boundaries, which causes the ballistic behavior. On the other hand, at large $t$, remaining grains are rare in metastable patterns. Avalanche flow keeps losing grains at avalanche boundaries, which also causes the ballistic behavior, resulting in $\alpha=1$.

In the odd case with $z_{c}=2 k+1$, the minimal number of grains at a stable site is $k$ unless empty. Assume that $z_{c}$ grains are at the rightmost site of an avalanche, say $(i, t)$, and its right neighboring site $(i+1, t+1)$ is occupied by $k$ grains. The site $(i+1, t+1)$ will topple only if it receives $k+1$ grains from the site $(i, t)$, or will not if it receives $k$ grains. Whether the site topples or not depends on the value of the toppling arrow $a(i, t)$. This randomness inherent to toppling arrows leads to the random walk behaviors of avalanche boundaries, i.e., $\alpha=\frac{1}{2}$. If more than $z_{c}$ grains topple at the rightmost site or if its right neighboring site is occupied by more than $k$ grains, the randomness of the alternative toppling bias is suppressed as in the aND with even $z_{c}$ values. The same scaling behavior is also observed in the pND with $p=\frac{1}{2}$, where the toppling bias is completely random. In the bND with no intrinsic randomness (the pND with $p=1$ ), we do not expect the random walk behaviors of avalanche boundaries, irrespective of the parity of the threshold value. The pND in the range of $\frac{1}{2}<p<1$ will be discussed with numerical results in Sec. IV.

\section{NUMERICAL RESULTS}

We performed extensive numerical simulations for all DSMs to confirm our conjecture for avalanche exponents in terms of the scar exponent, $\alpha_{\mathrm{sc}}\left(\alpha=\alpha_{\mathrm{sc}}\right.$ for nonAbelian cases), up to $L=T=2^{13}\left(T=2^{15}\right.$ or $L=2 T$ in some cases).

Using conventional successive slope techniques for at most $10^{9}$ avalanches in the steady state enough after the transient period, we measure the avalanche exponent set $\left\{\tau_{x}, D_{x}\right\}$ for all $x$ and the grain density exponent $\alpha$ ( $\alpha_{\mathrm{sc}}$ for Abelian cases). As discussed, spatially correlated scars are observed in all DSMs with the non-zero values of $\alpha_{\mathrm{sc}}$. The spatial correlations of metastable patterns are only observed in non-Abelian DSMs with the non-zero values of $\alpha$ (see Table $\llbracket$ and Fig. [3).

We calculate effective exponents from power-law distributions of various avalanche quantities, scaling relations among those quantities, and the algebraic decay of the grain occupation density in terms of following definitions:

$$
\begin{aligned}
\tau_{x}^{\mathrm{eff}}(x) & =-\frac{\ln P(x)-\ln P(x / m)}{\ln x-\ln (x / m)}, \\
D_{x}^{\mathrm{eff}}(t) & =\frac{\ln E[x \mid t]-\ln E[x \mid t / m]}{\ln t-\ln (t / m)}, \\
\alpha^{\mathrm{eff}}(t) & =-\frac{\ln \rho_{\theta}(t)-\ln \rho_{\theta}(t / m)}{\ln t-\ln (t / m)},
\end{aligned}
$$

as taking appropriate values of $m$. In order to get the reasonable resolution of effective exponents, we set $m=5$ for $\tau^{\text {eff }}$ and $\alpha^{\text {eff }}$ and $m=1$ for $D^{\text {eff }}$, respectively. The exponents, $\tau_{x}$ and $D_{x}\left(=\gamma_{x t}\right)$, are directly obtained by the linear fit of scaling regimes in power-law distribution functions and scaling relations. Scaling regimes can be identified either from the flat region of effective exponents or the systematic tendency as the system size increases. Regarding $\gamma_{y x}$, instead of the original definition, $E[y \mid x] \sim$ $x^{\gamma_{y x}}$, we make use of the modified definition, $E[y \mid x]=$ $x+C x^{\gamma_{y x}}$, when $y \geq x$ for each avalanche [25]. Due to $s \geq z_{c} t$ and $h \geq z_{c}$ by definition, we obtain $\gamma_{s t}$ and $\gamma_{h t}$ using $E[s \mid t]=z_{c} t+C t^{\gamma_{s t}}$ and $E[h \mid t]=z_{c}+C^{\prime} t^{\gamma_{h t}}$, respectively. The values of scaling exponents are listed in Table I, which confirm our conjecture by means of the avalanche flow analysis in Sec. III

In the aND, the scaling behaviors of grain occupation densities definitely depend on the parity of the threshold value, $z_{c}$, as shown in Fig. $4(\mathrm{a})$. For the even $z_{c}$ 

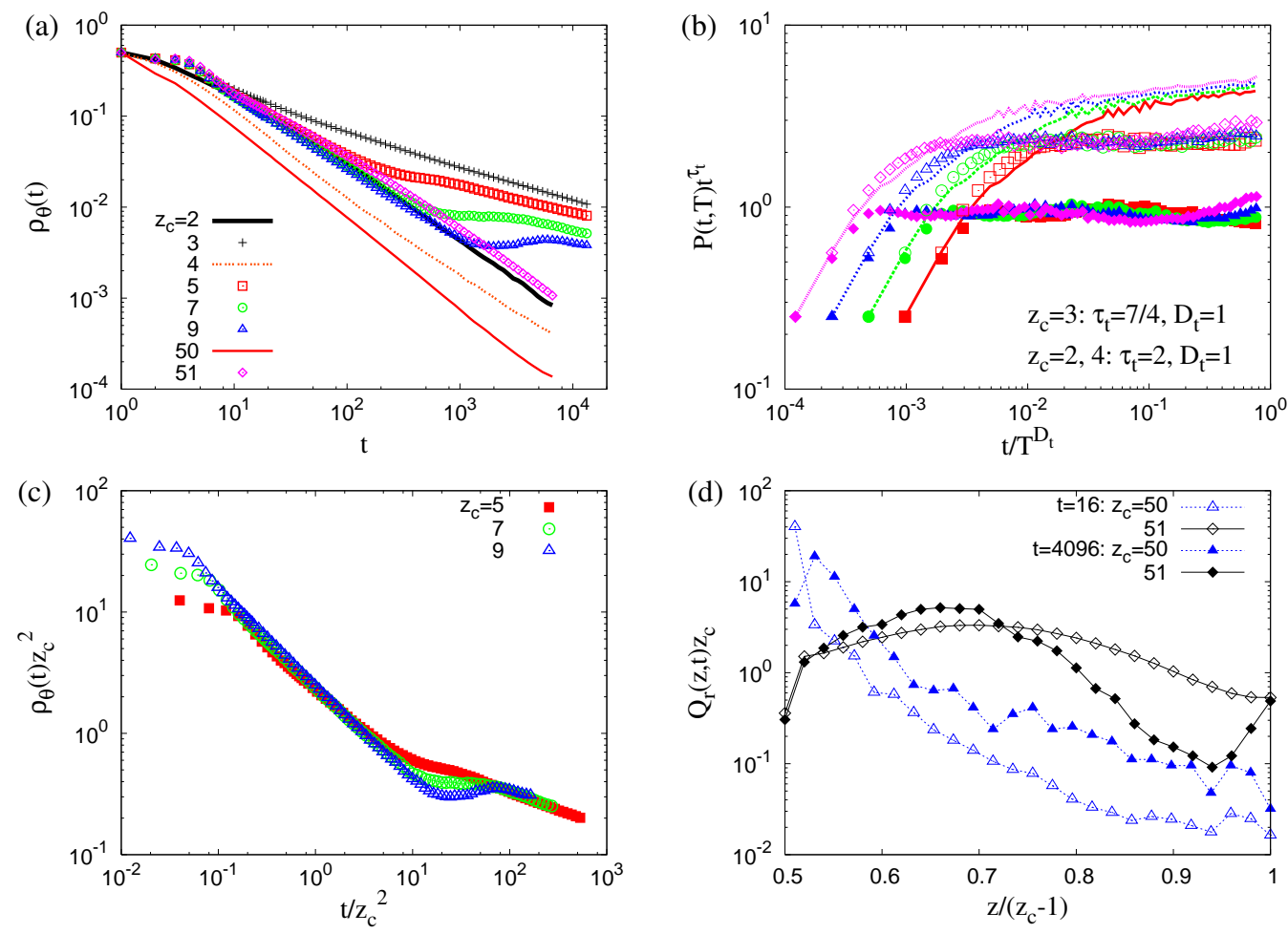

FIG. 4: (Color online) Numerical results of the aND for various $z_{c}$ values: (a) grain occupation densities $\rho_{\theta}(t)$ versus $t$ on a lattice with $T=2^{14}$ for $z_{c}=3,5,7$, and $9\left(T=2^{13}\right.$ otherwise) and $L=2 T$. (b) Finite-size scaling of $P(t, T)$ with the MF value $\left(\tau_{t}=2\right)$ for $z_{c}=2$ (lines) and $z_{c}=4$ (open symbols), and with the NS value $\left(\tau_{t}=7 / 4\right)$ at $z_{c}=3$ (filled symbols). Here, $T=L=2^{10}, 2^{11}, 2^{12}$, and $2^{13}$ from right to left and $D_{t}=1$ for all cases. (c) Crossover scaling of grain occupation densities for odd $z_{c}$ values by $\rho_{\theta}(t) z_{c}^{2}$ as a function of $t / z_{c}^{2}$, which are the same data as (a). (d) Rescaled grain distributions $Q_{r}(z, t)$ at two different layers, $t=16$ (open symbols) and 4096 (filled symbols) for $z_{c}=50(\triangle)$ and $51(\diamond)$ on a lattice with $T=2^{13}$ and $L=2 T$.
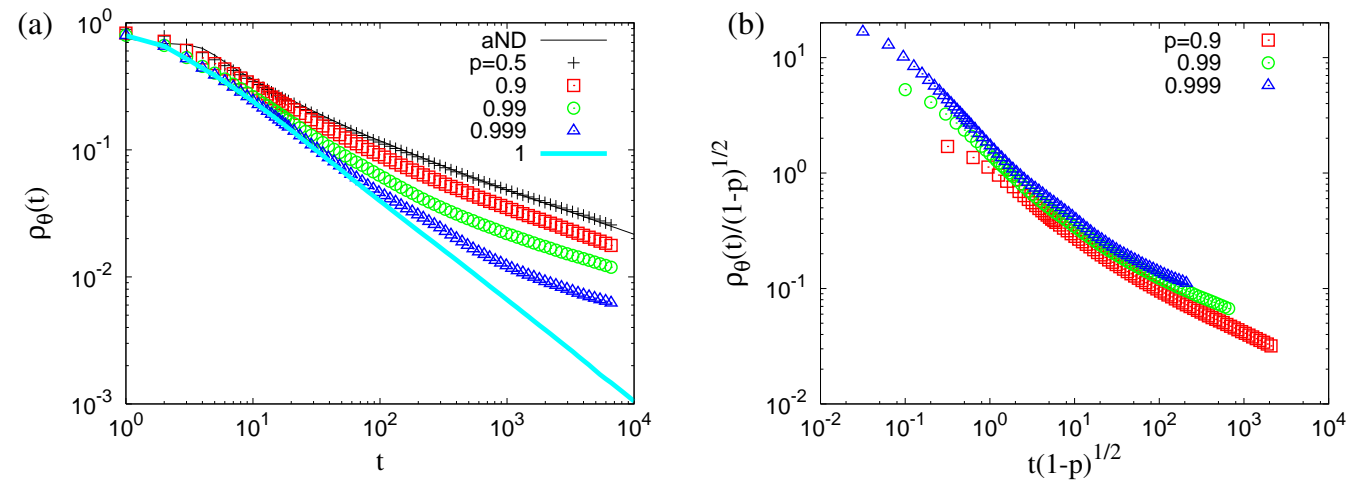

FIG. 5: (Color online) (a) Grain occupation densities $\rho_{\theta}(t)$ versus $t$ in the pND at a fixed $z_{c}=3$ as $p$ increases from 0.5 (the aND as the thin line at the top) to 1 (the bND as the thick line at the bottom). We used a lattice with $T=2^{13}$ and $L=2 T$. (b) Crossover scaling of grain occupation densities at $z_{c}=3$ by $\rho_{\theta}(t) /(1-p)^{1 / 2}$ as a function of $t(1-p)^{1 / 2}$.

values, the scaling regime of $\alpha^{\mathrm{eff}}(t)$ approaches 1 as $z_{c}$ increases (not shown). The value of $\alpha$ at $z_{c}=3$ appears to be $\frac{1}{2}$ within errors rather than 1 . Interestingly, for the larger odd $z_{c}$ values, we observe the crossover behavior from $\alpha^{\text {eff }}(t)=1$ (with logarithmic corrections) to $\frac{1}{2}$ at some "crossover layer," $t_{\times}$, which clearly increases as $z_{c}$ increases. We investigate the same parity effect on the universality class in terms of other effective exponents, $\tau_{s}^{\text {eff }}, \tau_{t}^{\text {eff }}$, and $D_{h}^{\text {eff }}$, for avalanche distributions (not shown). The effective exponents for $z_{c}=2$ and 4 approach MF values $\left(\tau_{s}=\frac{3}{2}, \tau_{t}=2\right.$, and $\left.D_{h}=0\right)$ or at least have the tendency to approach them. On the other hand, effective exponents at $z_{c}=3$ clearly deviate from MF values. We also confirm the finite-size scaling (FSS) 

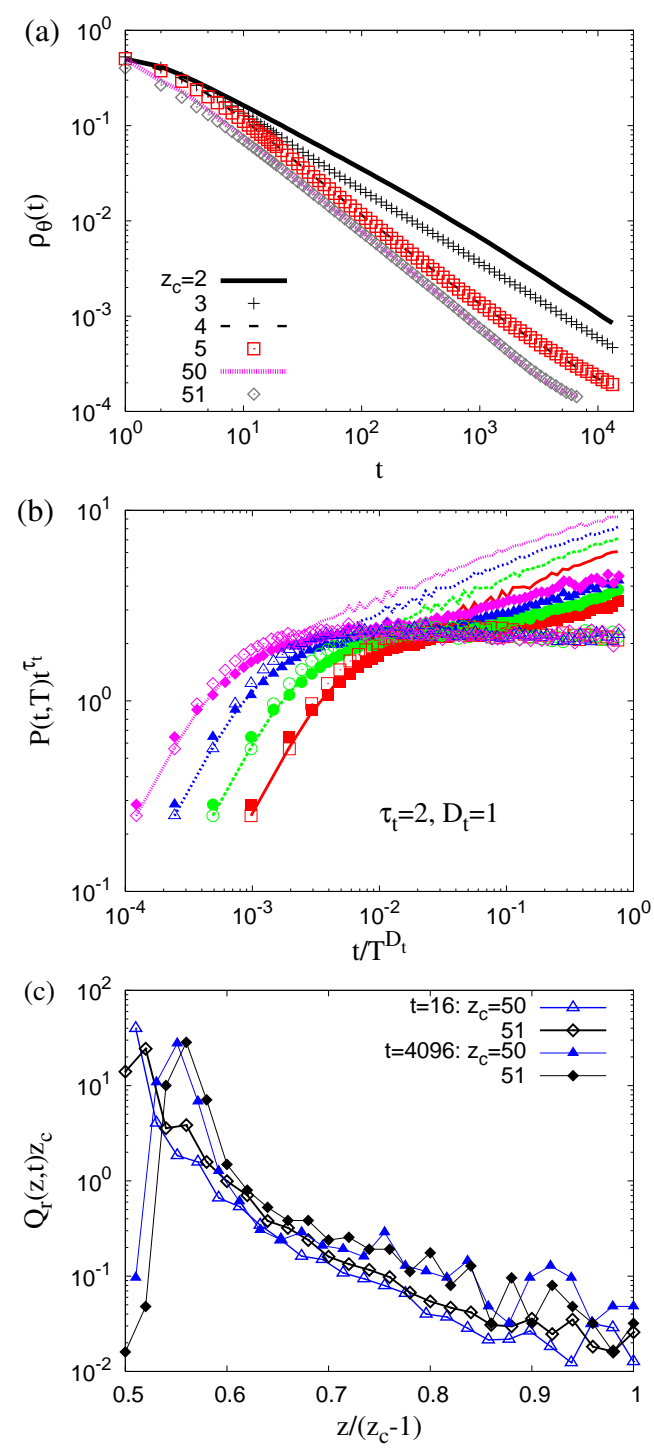

FIG. 6: (Color online) Numerical results of the bND for various $z_{c}$ values: (a) grain occupation densities $\rho_{\theta}(t)$ versus $t$ on a lattice with $T=2^{14}$ and $L=2 T\left(T=2^{13}\right.$ for $z_{c}=50$ and 51), where lines for $z_{c}=2,4$, and 50 are from top to bottom. (b) Finite-size scaling of $P(t, T)$ with MF values for $z_{c}=2$ (lines), $z_{c}=3$ (filled symbols), and $z_{c}=4$ (open symbols). Here $T=L=2^{10}, 2^{11}, 2^{12}$, and $2^{13}$ from right to left. (c) Rescaled grain distributions $Q_{r}(z, t)$ at two different layers, $t=16$ (open symbols) and 4096 (filled symbols) for $z_{c}=50$ $(\triangle)$ and $51(\diamond)$ on a lattice with $T=2^{13}$ and $L=2 T$.

behaviors of avalanche distributions with MF values for $z_{c}=2$ and 4 , and with NS values $\left(\tau_{s}=\frac{10}{7}, \tau_{t}=\frac{7}{4}\right.$, and $D_{h}=\frac{1}{4}$ ) at $z_{c}=3$. Figure 4(b) shows the successful FSS collapses of avalanche duration distributions.

Numerical data in the aND are averaged over a lot of avalanches (up to $10^{9}$ with only few numbers of ensemble for random initial configuration setups) in the steady state after the transient. Since toppling arrows, $\{a(i, t)\}$, are annealed enough by the random addition of grains in the steady state, it is guaranteed that all the results are
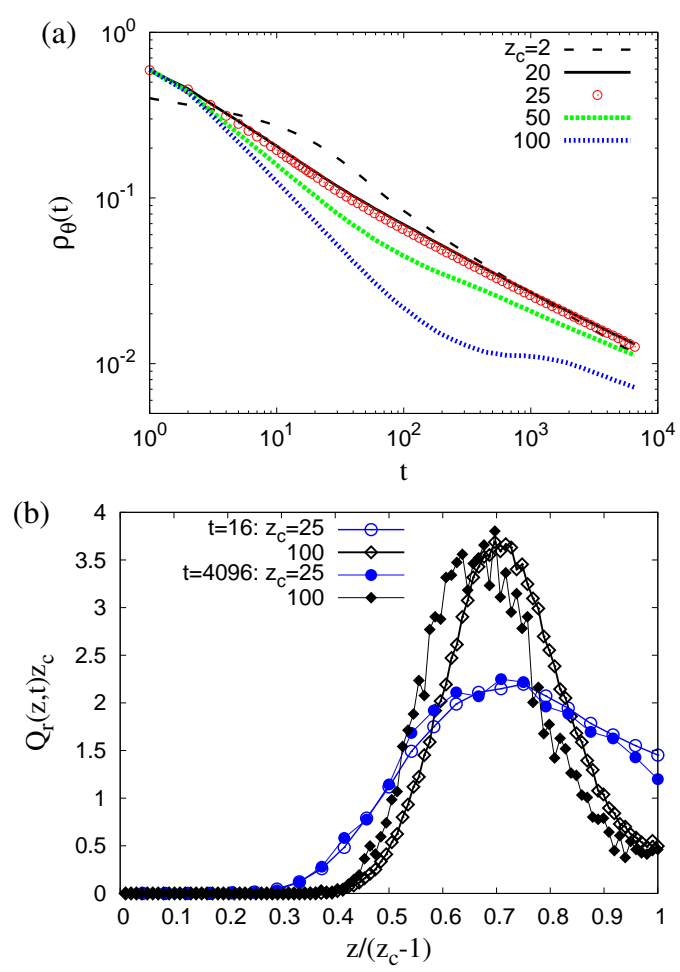

FIG. 7: (Color online) (a) Grain occupation densities $\rho_{\theta}(t)$ versus $t$ in the NS for various $z_{c}$ values (lines for $z_{c}=2$, 20, 50, and 100 from top to bottom at the right). We used a lattice with $T=2^{13}$ and $L=2 T$. (b) In particular to the cases for $z_{c}=25$ and 100, rescaled grain distributions, $Q_{r}(z, t)$, are measured at two different layers, $t=16$ (open symbols) and 4096 (filled symbols).

completely independent of initial conditions, which are also numerically confirmed.

Figure 4(c) shows the crossover scaling of grain occupation densities in the aND for odd $z_{c}$ values. It can be written as

$$
\rho_{\theta}(t)=t^{-1} g\left(t / t_{\times}\right)
$$

where the scaling function behaves as $g(x) \sim \mathcal{O}(1)$ for small $x$ and $g(x) \sim \sqrt{x}$ for large $x$. At small $x\left(t<t_{\times}\right)$, grain occupation densities are relatively high while the avalanche width is still small. Grains at avalanche bulk sites quickly diffuse to avalanche boundaries. The numbers of grains at avalanche boundaries tend to be larger than $z_{c}$ and thus make ballistic avalanche boundaries $(\alpha=1)$. As the grain occupation densities decay to become low at large $x\left(t>t_{\times}\right)$, the numbers of grains at avalanche boundaries tend to be $z_{c}$. Then the stochasticity of avalanche boundaries is disclosed $\left(\alpha=\frac{1}{2}\right)$. The value of $t_{\times}$indicates the crossover from the dense region to the sparse region of remaining grains. We present some naive test for the crossover scaling of $\rho_{\theta}$ using $t_{\times} \sim z_{c}^{2}$. Indeed, numerical data collapse pretty well for $z_{c}=5,7$, and 9, as shown in Fig. 4(c). This can be explained by an intuitive argument. As discussed in the last part of 
Sect.III the stochasticity inherent to toppling arrows reveals only in the case when the unstable boundary site, say, $(i, t)$ at the right boundary, has $z_{c}$ grains and its neighboring site at the next layer, say, $(i+1, t+1)$, is occupied by $k$ grains. Assuming that the grain distribution is uniform (to be confirmed), the probability of an occupied site having $k$ grains is about $z_{c}^{-1}$. If the unstable site has $z_{c}$ grains, it might have been occupied by $k$ or $k+1$ grains before receiving grains from the unstable neighboring site at the previous layer, whose probability is about $z_{c}^{-1}$. Then, the stochasticity in toppling arrows begins to appear when the expected number of such cases up to the layer $t$ becomes finite, i.e., $z_{c}^{-2} t \sim \mathcal{O}(1)$, which implies that $t_{\times} \sim z_{c}^{2}$.

In the aND at $z_{c}=51$, the value of $\alpha$ approaches 1 in Fig. 4(a), which may mislead to conclude no crossover for the larger $z_{c}$ values even if they are odd. We here pose the following question: How can we know whether or not any crossover behavior is observed at the sufficiently large layer? The value of $\alpha$ is not sufficient enough to answer the question. We need to investigate the grain distribution $Q(z, t)$ in Eq. (7) that contains the detailed information for metastable grain patterns. Due to the fact that the $Q(0, t)=1-\rho_{\theta}(t)$ becomes dominant as $t$ increases for non-Abelian DSMs, we consider the rescaled grain distribution as

$$
Q_{r}(z, t)=\frac{Q(z, t)}{1-Q(0, t)},
$$

where $Q_{r}(z, t)=0$ for $z \in\left\{1,2, \ldots,\left[z_{c} / 2\right]-1\right\}$.

We numerically find that $Q_{r}(z, t)$ is broad due to the stochastic nature for odd $z_{c}$ values. It is also found that it is peaked around at the value of $\left[z_{c} / 2\right]$ due to the deterministic nature for even $z_{c}$ values. Figure 4 (d) shows the broadness of $Q_{r}(z, t)$ for $z_{c}=51$ and the peaked structure of $Q_{r}(z, t)$ for $z_{c}=50$. This implies that the shape of the grain distribution is one of the good indicators to identify the universality class, as well as the grain density exponent in metastable patterns.

The effect of the quenched randomness in the aND is compared to that of the annealed randomness in the $\mathrm{pND}$. The scaling behaviors of $\rho_{\theta}(t)$ in the pND with $p=\frac{1}{2}$ for various $z_{c}$ values exhibit qualitatively the same behaviors as those in the aND (not shown). Since the scaling behavior of $\rho_{\theta}(t)$ in the aND $\left(\alpha=\frac{1}{2}\right)$ at $z_{c}=3$ is quite different from that in the $\operatorname{bND}(\alpha=1)$ at the same $z_{c}$ value, one can ask if another crossover behavior exists between them. At each value of $p$ close to 1 in the $\mathrm{pND}$, we are able to observe the crossover behavior of the grain occupation density from $\alpha=1$ to $\frac{1}{2}$ at some crossover layer, $t_{\times}$, as shown in Fig. F(a). We numerically find that the value of $t_{\times}$scales as $(1-p)^{-1 / 2}$, [see Fig. [5)(b)]. Such scaling is roughly explained by the following argument: the degree of stochasticity per toppling event is simply given by $1-p$. The total number of realizations up to the layer $t$ is on the order of $w(t) t \sim t^{2}$ for $t<t_{\times}$. Thus, the stochasticity begins to appear when $(1-p) t^{2} \sim \mathcal{O}(1)$, which implies that $t_{\times} \sim(1-p)^{-1 / 2}$.
Figure 6(a) shows the scaling behaviors of $\rho_{\theta}(t)$ in the bND for various $z_{c}$ values. The results imply the MF class with some logarithmic corrections to scaling for small $z_{c}$ values and without corrections for large $z_{c}$ values. For $z_{c}=2$ and $3, \alpha^{\text {eff }}(t)$ values are less than 1 even in the scaling regime (not shown), but the possibility to approach 1 cannot be excluded. Such deviation affects other scaling behaviors. We also measure the effective exponents of avalanche distributions and test the FSS collapse with MF values for $z_{c}=2,3$, and 4 [see Fig. [6(b)]. From our numerical observation, it is difficult to determine whether the cases of $z_{c}=2$ and 3 obey eventually the same FSS as the cases of $z_{c} \geq 4$ or not. Figure 6(c) shows that $Q_{r}(z, t)$ is peaked around at $\left[z_{c} / 2\right]$, consistent with the observation that avalanche boundaries behave ballistically, which implies again the MF class, independent of the parity of $z_{c}(\geq 4)$.

Next, we investigate another kind of crossover behaviors from the NS class to the MF class in the NS as $z_{c}$ increases up to 100 . This is motivated by Lübeck's work [21] that reported the crossover behavior between the undirected NS and the undirected cND for very large $z_{c}$ values (up to the order of $10^{3}$ ). In the undirected NS, if $m \geq z_{c}$ grains topple at an unstable site, its neighboring sites will receive grains of $\mathcal{O}(m)$ on average with the standard deviation of $\mathcal{O}(\sqrt{m})$. Thus, for sufficiently large $z_{c}$ values, the stochasticity in toppling rules is effectively suppressed and the system shows the deterministic universality class. It was claimed that universality classes can be determined in the context of the shape of grain distributions: the grain distribution is broad for the undirected stochastic class, while it is narrow with multiple peaks for the undirected deterministic class. In the similar sense, one can expect essentially the same crossover from the NS class to the MF class in the directed case as $z_{c}$ increases to the sufficiently large value, i.e., by suppressing fluctuations in $\tilde{\Delta}_{i \pm 1, t+1}$.

In Fig. 7(a), we observe two kinds of crossover behaviors of grain occupation densities in DSMs. One is from $\alpha=\frac{1}{2}$ (NS class) to 1 (MF class) as $z_{c}$ increases, as expected. The other is from $\alpha=1$ to $\frac{1}{2}$ at a fixed $z_{c}$ value as $t$ increases. Here the crossover layer, $t_{\times}$, depends on the $z_{c}$ value. We systematically observe that the shape of $Q_{r}(z, t)$ at $z_{c}=100$ is much narrower than that at $z_{c}=25$ for any layer $t$ [see Fig. 7(b)]. It implies the MF class for much larger $z_{c}$ values, which is hardly confirmed in numerical simulations.

Finally, we discuss the crossover mechanism from the MF class to the NS class at a fixed $z_{c}$ value for the directed case. The mechanism is different from that for the undirected case. The numbers of toppled grains at unstable sites on the top layer $t=0$ are always $z_{c}$. Then, $Q(z, t=1)$ has the normal distribution with the mean value of $z_{c} / 2$ and the standard deviation on the order of $\sqrt{z_{c}}$, as well as an additional peak around $z_{c}-1$. The latter peak is due to the sites that have received grains both from the neighboring sites on the top layer, but are still stable. This peaked structure implies the MF 
class. Inevitable fluctuations around two peaks in the $Q(z, t)$ at small $t$ propagate and accumulate through layers, and finally result in the broad distribution without any distinguished peaks. If the value of $z_{c}$ was sufficiently large enough to exclude any fluctuations (approximately the cND), the MF behavior would be clearly observed without any crossover behaviors. In other words, for the finite $z_{c}$ values, there always exists crossovers from the MF class to the NS class at some finite crossover layer.

\section{SUMMARY}

We studied both non-Abelian stochastic (NS) and mean-field (MF) universality classes and crossover behaviors between two classes in various non-Abelian directed sandpile models (DSMs) on a two-dimensional tilted lattice. The broken Abelian symmetry induces spatially long-range correlations in metastable patterns. The universality class of avalanche dynamics can be identified by means of the grain density exponent in metastable patterns: $\alpha=\frac{1}{2}$ for the NS class and $\alpha=1$ for the MF class. Due to some ambiguity of the non-Abelian deterministic toppling rule against the given lattice structure, we considered the toppling bias for the last grain. We found that the parity of the threshold value, $z_{c}$, might change the universality class. Using extensive numerical simulations, we confirmed that MF behaviors are well observed in the aND for even $z_{c}$ values and in the bND for any $z_{c}$ values as $z_{c}$ increases. For odd $z_{c}$ values, the toppling randomness in the aND and in the pND with $p=\frac{1}{2}$ becomes relevant, so that they belong to the NS class.
In the aND and the pND with $p=\frac{1}{2}$ for large odd $z_{c}$ values, we numerically observed crossover behaviors from $\alpha=1$ to $\frac{1}{2}$ at some crossover layer, $t_{\times}$. The crossover layer is a function of the $z_{c}$ value and the partial bias, $p$, in the pND. Since the grain density exponent is not sufficient to determine the universality class under some circumstances, we suggest checking the broadness of the grain distribution in metastable patterns as well. Here the broadness originates from the stochastic nature in toppling rules. As in the undirected case, we also observed that the NS class undergoes the crossover to the $\mathrm{MF}$ class as $z_{c}$ increases. At a fixed $z_{c}$ value, another crossover is found from the MF class to the NS class at a finite value of $t_{\times}$.

In conclusion, we emphasize that one should be very careful to set the toppling bias and the threshold value for some appropriate minimal model of self-organized criticality in order to explain experimental results. It is because the naive setting of parameters may mislead to either unexpected universality classes or crossover behaviors. Finally, it would be worthwhile to extend our various tests towards some other lattice structures, to clarify the crossover scaling issues more analytically, and to test other types of non-Abelian DSMs, such as sticky sandpiles and sandpiles with bulk dissipation [26].

\section{ACKNOWLEDGMENTS}

This work was supported by the BK21 project and by NAP of Korea Research Council of Fundamental Science and Technology (MH). We would like to thank D. Dhar for reading our paper and giving valuable comments on it as well as suggesting further works.
[1] K. Christensen and N.R. Moloney, Complexity and Criticality (Imperial College Press, London, 2005).

[2] M.E.J. Newman, Contemp. Phys. 46, 323 (2005).

[3] J.S. Urbach, R.C. Madison, and J.T. Markert, Phys. Rev. Lett. 75, 276 (1995); K.-S. Ryu, H. Akinaga, and S.-C. Shin, Nat. Phys. 3, 547 (2007).

[4] P. Bak, C. Tang, and K. Wiesenfeld, Phys. Rev. Lett. 59, 381 (1987).

[5] H.J. Jensen, Self-Organized Criticality (Cambridge University Press, Cambridge, 1998).

[6] D. Dhar, Physica A 369, 29 (2006).

[7] Y.-C. Zhang, Phys. Rev. Lett. 63, 470 (1989).

[8] S.S. Manna, J. Phys. A 24, L363 (1991).

[9] A. Ben-Hur and O. Biham, Phys. Rev. E 53, R1317 (1996).

[10] S. Lübeck and K.D. Usadel, Phys. Rev. E 55, 4095 (1997).

[11] S. Lübeck, Phys. Rev. E 56, 1590 (1997).

[12] E. Milshtein, O. Biham, and S. Solomon, Phys. Rev. E 58, 303 (1998).

[13] R. Dickman and J.M.M. Campelo, Phys. Rev. E 67, 066111 (2003).

[14] D. Dhar and R. Ramaswamy, Phys. Rev. Lett. 63, 1659 (1989).
[15] R. Pastor-Satorras and A. Vespignani, J. Phys. A 33, L33 (2000); Phys. Rev. E 62, 6195 (2000).

[16] M. Paczuski and K.E. Bassler, Phys. Rev. E 62, 5347 (2000); cond-mat/0005340v2.

[17] M. Kloster, S. Maslov, and C. Tang, Phys. Rev. E 63, 026111 (2001).

[18] D. Hughes and M. Paczuski, Phys. Rev. Lett. 88, 054302 (2002); cond-mat/0105408v1.

[19] G.-J. Pan et al., Phys. Lett. A 338, 163 (2005).

[20] H.-H. Jo and M. Ha, Phys. Rev. Lett. 101, 218001 (2008).

[21] S. Lübeck, Phys. Rev. E 62, 6149 (2000).

[22] H. Hinrichsen, Adv. Phys. 49, 815 (2000).

[23] For some cases, the deviation from our conjecture may be attributed to logarithmic corrections to scaling as well as the finite-size effect.

[24] In general, on a $(d+1)$-dimensional lattice, $a \sim t w^{d}$ yielding $D_{a}=1+d D_{w}$. In case of $d \geq d_{u}$, where $d_{u}=2$ is the upper critical dimension of DSMs, $d D_{w}=1$. This is consistent with our MF results, i.e., $D_{w}=1, d=1$.

[25] A. Chessa, H.E. Stanley, A. Vespignani, and S. Zapperi, Phys. Rev. E 59, R12 (1999).

[26] D. Dhar (private communication). 